\title{
Author Correction: High-pressure versus low-pressure home non-invasive positive pressure ventilation with built-in software in patients with stable hypercapnic COPD: a pilot study
}

Luqian Zhou ${ }^{1}$, Lili Guan ${ }^{1}$, Weiliang Wu${ }^{1}$, Xiaoying Li ${ }^{2}$, Xin Chen ${ }^{3}$, Bingpeng Guo ${ }^{1}$, Yating Huo ${ }^{1}$, Jiawen $\mathrm{Xu}^{1}$, Yuqiong Yang ${ }^{1} \&$ Rongchang $\mathrm{Chen}^{1}$

Correction to: Scientific Reports https://doi.org/10.1038/s41598-017-17142-2, published online 01 December 2017

In the original version of this Article, Luqian Zhou, and not Rongchang Chen, was listed as the corresponding author. Correspondence and request for materials should be addressed to Rongchang Chen at chenrcstatekeylab@ gmail.com.

In addition, the order of the author names was incorrectly given as 'Rongchang Chen, Lili Guan, Weiliang Wu, Xiaoying Li, Xin Chen, Bingpeng Guo, Yating Huo, Jiawen Xu, Yuqiong Yang \& Luqian Zhou'

Finally,

"Rongchang Chen, Lili Guan, Weiliang Wu and Xiaoying Li contributed equally to this work."

now reads:

“Luqian Zhou, Lili Guan, Weiliang Wu and Xiaoying Li contributed equally to this work."

These errors have now been corrected in the HTML and PDF versions of this Article.

(i) Open Access This article is licensed under a Creative Commons Attribution 4.0 International License, which permits use, sharing, adaptation, distribution and reproduction in any medium or format, as long as you give appropriate credit to the original author(s) and the source, provide a link to the Creative Commons license, and indicate if changes were made. The images or other third party material in this article are included in the article's Creative Commons license, unless indicated otherwise in a credit line to the material. If material is not included in the article's Creative Commons license and your intended use is not permitted by statutory regulation or exceeds the permitted use, you will need to obtain permission directly from the copyright holder. To view a copy of this license, visit http://creativecommons.org/licenses/by/4.0/.

(C) The Author(s) 2018

${ }^{1}$ State Key Laboratory of Respiratory Disease, Guangzhou Institute of Respiratory Disease, the First Affiliated Hospital of Guangzhou Medical University, Guangzhou, China. ${ }^{2}$ The First Affiliated Hospital/School of Clinical Medicine of Guangdong Pharmaceutical University, Guangzhou, China. ${ }^{3}$ ZhuJiang Hospital of Southern Medical University, Guangzhou, China. Luqian Zhou, Lili Guan, Weiliang Wu and Xiaoying Li contributed equally to this work. Correspondence and requests for materials should be addressed to R.C. (email: chenrcstatekeylab@gmail.com) 